\title{
On the strategies for incorporating nanosilica aqueous dispersion in the synthesis of waterborne polyurethane/silica nanocomposites: Effects on morphology and properties
}

\author{
Pablo J. Peruzzo ${ }^{\mathrm{a}, *}$, Pablo S. Anbinder ${ }^{\mathrm{b}}$, Francisco M. Pardini ${ }^{\mathrm{c}}$, Oscar R. Pardini ${ }^{\mathrm{a}, \mathrm{c}}$, \\ Tomas S. Plivelic ${ }^{\mathrm{d}}$, Javier I. Amalvy ${ }^{\mathrm{a}, \mathrm{c}}$ \\ a Instituto de Investigaciones Fisicoquímicas Teóricas y Aplicadas (INIFTA: UNLP-CONICET CCT La Plata), Universidad Nacional de La Plata, Diag. 113 y \\ 64CC 16 Suc. 4, B1904DPI La Plata, Argentina \\ b Instituto de Física de Materiales Tandil (IFIMAT), CIFICEN, Consejo Nacional de Investigaciones Científicas y Técnicas (CONICET) y Universidad del Centro \\ de la Provincia de Buenos Aires, Pinto 399, B7000GHG Tandil, Argentina \\ ' Centro de Investigación y Desarrollo en Tecnología de Pinturas (CIDEPINT: CICPBA-CONICET CCT La Plata), Av. 52 e/121 y 122. B1900AYB La Plata, \\ Argentina \\ ${ }^{\mathrm{d}}$ MAX IV Laboratory, Lund University, P.O. Box 118, 22100 Lund, Sweden
}

\section{Keywords:}

Nanocomposites

Polyurethane/nanosilica

Waterborne dispersions

\begin{abstract}
A B S T R A C T
In this work the synthesis of waterborne polyurethane/nanosilica nanocomposites by using two different strategies is presented, starting from a vinyl terminated polyurethane prepolymer (PUP) based on isophorone diisocyanate and polypropylene glycol, and varying the nanosilica content. In one case, the PUP was dispersed in an aqueous solution containing glycerol-functionalized colloidal nanosilica particles and the dispersion was further polymerized; in the other case, the PUP was dispersed in an aqueous media, then colloidal nanosilica was added to the dispersion and then polymerized. A physical mixture was also prepared for comparative purpose. Films were prepared by casting of the aqueous dispersion. The morphology of the dispersions and films depended on the incorporation route of nanoparticles as was observed by SAXS, SEM and TEM. While the blends had significantly less hydrogen bonding between the hard segments of the PU and nanosilica particles, samples prepared by the two different methods proposed in this work showed an strong interaction between both materials in agreement with FTIR and DSC results. Synthesis pathway plays an important role in order to obtain high performance waterborne polyurethane/nanosilica composites, since final properties of the films also depended on the nanoparticle incorporation strategy.
\end{abstract}

\section{Introduction}

Organic/inorganic composite materials have been extensively studied for a long time. When inorganic phases in organic/inorganic composites become nanosized, they are called nanocomposites. In polymer nanocomposites the polymer chains are confined to nanoscale dimensions $(1-10 \mathrm{~nm})$. These materials have unusual properties which cannot be obtained simply by co-mixing the polymeric component with the inorganic phase at the macroscopic level [1-4]. However, the properties of nanocomposites strongly depend

\footnotetext{
* Corresponding author. Tel.: +54 $2214257291 / 7434$ (int: 115); fax: +54 2214254642 .

E-mail address: pjperuzzo@inifta.unl p.edu.ar (P.J. Peruzzo).
}

on the organic matrix, inorganic nanofiller and the way in which they are prepared [5-7].

Polyurethane/silica ( $\mathrm{PU} /$ silica) nanocomposites is one of the combinations that have attracted substantial academic and industrial interest in the last 20 years. In fact, among the numerous organic/inorganic nanocomposites, $\mathrm{PU} /$ silica nanocomposites have received much attention in recent years and they have been employed in a variety of applications [8,9]. The incorporation of nanosilica to the polyurethane matrix provide films with improved properties like increased indentation hardness, high resistance to whitening and high permeability to water vapor [10]. These materials are useful also as tough, abrasion-resistant coatings with increasing friction coefficient and reduced tackiness, with no change in gloss [11].

According to the starting materials and processing techniques $\mathrm{PU} /$ silica nanocomposites are prepared by three general methods 


\title{
On the strategies for incorporating nanosilica aqueous dispersion in the synthesis of waterborne polyurethane/silica nanocomposites: Effects on morphology and properties
}

\author{
Pablo J. Peruzzo ${ }^{\mathrm{a}, *}$, Pablo S. Anbinder ${ }^{\mathrm{b}}$, Francisco M. Pardini ${ }^{\mathrm{c}}$, Oscar R. Pardini ${ }^{\mathrm{a}, \mathrm{c}}$, \\ Tomas S. Plivelic ${ }^{\mathrm{d}}$, Javier I. Amalvy ${ }^{\mathrm{a}, \mathrm{c}}$ \\ a Instituto de Investigaciones Fisicoquímicas Teóricas y Aplicadas (INIFTA: UNLP_CONICET CCT La Plata), Universidad Nacional de La Plata, Diag. 113 y \\ 64CC 16 Suc. 4, B1904DPI La Plata, Argentina \\ b Instituto de Física de Materiales Tandil (IFIMAT), CIFICEN, Consejo Nacional de Investigaciones Científicas y Técnicas (CONICET) y Universidad del Centro \\ de la Provincia de Buenos Aires, Pinto 399, B7000GHG Tandil, Argentina \\ 'Centro de Investigación y Desarrollo en Tecnología de Pinturas (CIDEPINT: CICPBA-CONICET CCT La Plata), Av. 52 e/121 y 122. B1900AYB La Plata, \\ Argentina \\ ${ }^{\mathrm{d}}$ MAX IV Laboratory, Lund University, P.O. Box 118, 22100 Lund, Sweden
}

\section{A B S T R A C T}

In this work the synthesis of waterborne polyurethane/nanosilica nanocomposites by using two different strategies is presented, starting from a vinyl terminated polyurethane prepolymer (PUP) based on isophorone diisocyanate and polypropylene glycol, and varying the nanosilica content. In one case, the PUP was dispersed in an aqueous solution containing glycerol-functionalized colloidal nanosilica particles and the dispersion was further polymerized; in the other case, the PUP was dispersed in an aqueous media, then colloidal nanosilica was added to the dispersion and then polymerized. A physical mixture was also prepared for comparative purpose. Films were prepared by casting of the aqueous dispersion.

Keywords:

Nanocomposites

Polyurethane/nanosilica

Waterborne dispersions
The morphology of the dispersions and films depended on the incorporation route of nanoparticles as was observed by SAXS, SEM and TEM. While the blends had significantly less hydrogen bonding between the hard segments of the PU and nanosilica particles, samples prepared by the two different methods proposed in this work showed an strong interaction between both materials in agreement with FTIR and DSC results. Synthesis pathway plays an important role in order to obtain high performance waterborne polyurethane/nanosilica composites, since final properties of the films also depended on the nanoparticle incorporation strategy.

\section{Introduction}

Organic/inorganic composite materials have been extensively studied for a long time. When inorganic phases in organic/inorganic composites become nanosized, they are called nanocomposites. In polymer nanocomposites the polymer chains are confined to nanoscale dimensions $(1-10 \mathrm{~nm})$. These materials have unusual properties which cannot be obtained simply by co-mixing the polymeric component with the inorganic phase at the macroscopic level [1-4]. However, the properties of nanocomposites strongly depend

* Corresponding author. Tel.: +54 $2214257291 / 7434$ (int: 115); fax: +54221 4254642

E-mail address: pjperuzzo@inifta.unl p.edu.ar (P.J. Peruzzo). on the organic matrix, inorganic nanofiller and the way in which they are prepared [5-7].

Polyurethane/silica (PU/silica) nanocomposites is one of the combinations that have attracted substantial academic and industrial interest in the last 20 years. In fact, among the numerous organic/inorganic nanocomposites, $\mathrm{PU} /$ silica nanocomposites have received much attention in recent years and they have been employed in a variety of applications [8,9]. The incorporation of nanosilica to the polyurethane matrix provide films with improved properties like increased indentation hardness, high resistance to whitening and high permeability to water vapor [10]. These materials are useful also as tough, abrasion-resistant coatings with increasing friction coefficient and reduced tackiness, with no change in gloss [11].

According to the starting materials and processing techniques $\mathrm{PU} /$ silica nanocomposites are prepared by three general methods 


\title{
On the strategies for incorporating nanosilica aqueous dispersion in the synthesis of waterborne polyurethane/silica nanocomposites: Effects on morphology and properties
}

\author{
Pablo J. Peruzzo ${ }^{\mathrm{a}, *}$, Pablo S. Anbinder ${ }^{\mathrm{b}}$, Francisco M. Pardini ${ }^{\mathrm{c}}$, Oscar R. Pardini ${ }^{\mathrm{a}, \mathrm{c}}$, \\ Tomas S. Plivelic ${ }^{\mathrm{d}}$, Javier I. Amalvy ${ }^{\mathrm{a}, \mathrm{c}}$ \\ a Instituto de Investigaciones Fisicoquímicas Teóricas y Aplicadas (INIFTA: UNLP_CONICET CCT La Plata), Universidad Nacional de La Plata, Diag. 113 y \\ 64CC 16 Suc. 4, B1904DPI La Plata, Argentina \\ b Instituto de Física de Materiales Tandil (IFIMAT), CIFICEN, Consejo Nacional de Investigaciones Científicas y Técnicas (CONICET) y Universidad del Centro \\ de la Provincia de Buenos Aires, Pinto 399, B7000GHG Tandil, Argentina \\ 'Centro de Investigación y Desarrollo en Tecnología de Pinturas (CIDEPINT: CICPBA-CONICET CCT La Plata), Av. 52 e/121 y 122. B1900AYB La Plata, \\ Argentina \\ ${ }^{\mathrm{d}}$ MAX IV Laboratory, Lund University, P.O. Box 118, 22100 Lund, Sweden
}

\section{A B S T R A C T}

In this work the synthesis of waterborne polyurethane/nanosilica nanocomposites by using two different strategies is presented, starting from a vinyl terminated polyurethane prepolymer (PUP) based on isophorone diisocyanate and polypropylene glycol, and varying the nanosilica content. In one case, the PUP was dispersed in an aqueous solution containing glycerol-functionalized colloidal nanosilica particles and the dispersion was further polymerized; in the other case, the PUP was dispersed in an aqueous media, then colloidal nanosilica was added to the dispersion and then polymerized. A physical mixture was also prepared for comparative purpose. Films were prepared by casting of the aqueous dispersion.

Keywords:

Nanocomposites

Polyurethane/nanosilica

Waterborne dispersions
The morphology of the dispersions and films depended on the incorporation route of nanoparticles as was observed by SAXS, SEM and TEM. While the blends had significantly less hydrogen bonding between the hard segments of the PU and nanosilica particles, samples prepared by the two different methods proposed in this work showed an strong interaction between both materials in agreement with FTIR and DSC results. Synthesis pathway plays an important role in order to obtain high performance waterborne polyurethane/nanosilica composites, since final properties of the films also depended on the nanoparticle incorporation strategy.

\section{Introduction}

Organic/inorganic composite materials have been extensively studied for a long time. When inorganic phases in organic/inorganic composites become nanosized, they are called nanocomposites. In polymer nanocomposites the polymer chains are confined to nanoscale dimensions $(1-10 \mathrm{~nm})$. These materials have unusual properties which cannot be obtained simply by co-mixing the polymeric component with the inorganic phase at the macroscopic level [1-4]. However, the properties of nanocomposites strongly depend

* Corresponding author. Tel.: +54 $2214257291 / 7434$ (int: 115); fax: +54221 4254642

E-mail address: pjperuzzo@inifta.unl p.edu.ar (P.J. Peruzzo). on the organic matrix, inorganic nanofiller and the way in which they are prepared [5-7].

Polyurethane/silica (PU/silica) nanocomposites is one of the combinations that have attracted substantial academic and industrial interest in the last 20 years. In fact, among the numerous organic/inorganic nanocomposites, $\mathrm{PU} /$ silica nanocomposites have received much attention in recent years and they have been employed in a variety of applications [8,9]. The incorporation of nanosilica to the polyurethane matrix provide films with improved properties like increased indentation hardness, high resistance to whitening and high permeability to water vapor [10]. These materials are useful also as tough, abrasion-resistant coatings with increasing friction coefficient and reduced tackiness, with no change in gloss [11].

According to the starting materials and processing techniques $\mathrm{PU} /$ silica nanocomposites are prepared by three general methods 


\title{
On the strategies for incorporating nanosilica aqueous dispersion in the synthesis of waterborne polyurethane/silica nanocomposites: Effects on morphology and properties
}

\author{
Pablo J. Peruzzo ${ }^{\mathrm{a}, *}$, Pablo S. Anbinder ${ }^{\mathrm{b}}$, Francisco M. Pardini ${ }^{\mathrm{c}}$, Oscar R. Pardini ${ }^{\mathrm{a}, \mathrm{c}}$, \\ Tomas S. Plivelic ${ }^{\mathrm{d}}$, Javier I. Amalvy ${ }^{\mathrm{a}, \mathrm{c}}$ \\ a Instituto de Investigaciones Fisicoquímicas Teóricas y Aplicadas (INIFTA: UNLP_CONICET CCT La Plata), Universidad Nacional de La Plata, Diag. 113 y \\ 64CC 16 Suc. 4, B1904DPI La Plata, Argentina \\ b Instituto de Física de Materiales Tandil (IFIMAT), CIFICEN, Consejo Nacional de Investigaciones Científicas y Técnicas (CONICET) y Universidad del Centro \\ de la Provincia de Buenos Aires, Pinto 399, B7000GHG Tandil, Argentina \\ 'Centro de Investigación y Desarrollo en Tecnología de Pinturas (CIDEPINT: CICPBA-CONICET CCT La Plata), Av. 52 e/121 y 122. B1900AYB La Plata, \\ Argentina \\ ${ }^{\mathrm{d}}$ MAX IV Laboratory, Lund University, P.O. Box 118, 22100 Lund, Sweden
}

\section{A B S T R A C T}

In this work the synthesis of waterborne polyurethane/nanosilica nanocomposites by using two different strategies is presented, starting from a vinyl terminated polyurethane prepolymer (PUP) based on isophorone diisocyanate and polypropylene glycol, and varying the nanosilica content. In one case, the PUP was dispersed in an aqueous solution containing glycerol-functionalized colloidal nanosilica particles and the dispersion was further polymerized; in the other case, the PUP was dispersed in an aqueous media, then colloidal nanosilica was added to the dispersion and then polymerized. A physical mixture was also prepared for comparative purpose. Films were prepared by casting of the aqueous dispersion.

Keywords:

Nanocomposites

Polyurethane/nanosilica

Waterborne dispersions
The morphology of the dispersions and films depended on the incorporation route of nanoparticles as was observed by SAXS, SEM and TEM. While the blends had significantly less hydrogen bonding between the hard segments of the PU and nanosilica particles, samples prepared by the two different methods proposed in this work showed an strong interaction between both materials in agreement with FTIR and DSC results. Synthesis pathway plays an important role in order to obtain high performance waterborne polyurethane/nanosilica composites, since final properties of the films also depended on the nanoparticle incorporation strategy.

\section{Introduction}

Organic/inorganic composite materials have been extensively studied for a long time. When inorganic phases in organic/inorganic composites become nanosized, they are called nanocomposites. In polymer nanocomposites the polymer chains are confined to nanoscale dimensions $(1-10 \mathrm{~nm})$. These materials have unusual properties which cannot be obtained simply by co-mixing the polymeric component with the inorganic phase at the macroscopic level [1-4]. However, the properties of nanocomposites strongly depend

* Corresponding author. Tel.: +54 $2214257291 / 7434$ (int: 115); fax: +54221 4254642

E-mail address: pjperuzzo@inifta.unl p.edu.ar (P.J. Peruzzo). on the organic matrix, inorganic nanofiller and the way in which they are prepared [5-7].

Polyurethane/silica (PU/silica) nanocomposites is one of the combinations that have attracted substantial academic and industrial interest in the last 20 years. In fact, among the numerous organic/inorganic nanocomposites, $\mathrm{PU} /$ silica nanocomposites have received much attention in recent years and they have been employed in a variety of applications [8,9]. The incorporation of nanosilica to the polyurethane matrix provide films with improved properties like increased indentation hardness, high resistance to whitening and high permeability to water vapor [10]. These materials are useful also as tough, abrasion-resistant coatings with increasing friction coefficient and reduced tackiness, with no change in gloss [11].

According to the starting materials and processing techniques $\mathrm{PU} /$ silica nanocomposites are prepared by three general methods 


\title{
On the strategies for incorporating nanosilica aqueous dispersion in the synthesis of waterborne polyurethane/silica nanocomposites: Effects on morphology and properties
}

\author{
Pablo J. Peruzzo ${ }^{\mathrm{a}, *}$, Pablo S. Anbinder ${ }^{\mathrm{b}}$, Francisco M. Pardini ${ }^{\mathrm{c}}$, Oscar R. Pardini ${ }^{\mathrm{a}, \mathrm{c}}$, \\ Tomas S. Plivelic ${ }^{\mathrm{d}}$, Javier I. Amalvy ${ }^{\mathrm{a}, \mathrm{c}}$ \\ a Instituto de Investigaciones Fisicoquímicas Teóricas y Aplicadas (INIFTA: UNLP_CONICET CCT La Plata), Universidad Nacional de La Plata, Diag. 113 y \\ 64CC 16 Suc. 4, B1904DPI La Plata, Argentina \\ b Instituto de Física de Materiales Tandil (IFIMAT), CIFICEN, Consejo Nacional de Investigaciones Científicas y Técnicas (CONICET) y Universidad del Centro \\ de la Provincia de Buenos Aires, Pinto 399, B7000GHG Tandil, Argentina \\ 'Centro de Investigación y Desarrollo en Tecnología de Pinturas (CIDEPINT: CICPBA-CONICET CCT La Plata), Av. 52 e/121 y 122. B1900AYB La Plata, \\ Argentina \\ ${ }^{\mathrm{d}}$ MAX IV Laboratory, Lund University, P.O. Box 118, 22100 Lund, Sweden
}

\section{A B S T R A C T}

In this work the synthesis of waterborne polyurethane/nanosilica nanocomposites by using two different strategies is presented, starting from a vinyl terminated polyurethane prepolymer (PUP) based on isophorone diisocyanate and polypropylene glycol, and varying the nanosilica content. In one case, the PUP was dispersed in an aqueous solution containing glycerol-functionalized colloidal nanosilica particles and the dispersion was further polymerized; in the other case, the PUP was dispersed in an aqueous media, then colloidal nanosilica was added to the dispersion and then polymerized. A physical mixture was also prepared for comparative purpose. Films were prepared by casting of the aqueous dispersion.

Keywords:

Nanocomposites

Polyurethane/nanosilica

Waterborne dispersions
The morphology of the dispersions and films depended on the incorporation route of nanoparticles as was observed by SAXS, SEM and TEM. While the blends had significantly less hydrogen bonding between the hard segments of the PU and nanosilica particles, samples prepared by the two different methods proposed in this work showed an strong interaction between both materials in agreement with FTIR and DSC results. Synthesis pathway plays an important role in order to obtain high performance waterborne polyurethane/nanosilica composites, since final properties of the films also depended on the nanoparticle incorporation strategy.

\section{Introduction}

Organic/inorganic composite materials have been extensively studied for a long time. When inorganic phases in organic/inorganic composites become nanosized, they are called nanocomposites. In polymer nanocomposites the polymer chains are confined to nanoscale dimensions $(1-10 \mathrm{~nm})$. These materials have unusual properties which cannot be obtained simply by co-mixing the polymeric component with the inorganic phase at the macroscopic level [1-4]. However, the properties of nanocomposites strongly depend

* Corresponding author. Tel.: +54 $2214257291 / 7434$ (int: 115); fax: +54221 4254642

E-mail address: pjperuzzo@inifta.unl p.edu.ar (P.J. Peruzzo). on the organic matrix, inorganic nanofiller and the way in which they are prepared [5-7].

Polyurethane/silica (PU/silica) nanocomposites is one of the combinations that have attracted substantial academic and industrial interest in the last 20 years. In fact, among the numerous organic/inorganic nanocomposites, $\mathrm{PU} /$ silica nanocomposites have received much attention in recent years and they have been employed in a variety of applications [8,9]. The incorporation of nanosilica to the polyurethane matrix provide films with improved properties like increased indentation hardness, high resistance to whitening and high permeability to water vapor [10]. These materials are useful also as tough, abrasion-resistant coatings with increasing friction coefficient and reduced tackiness, with no change in gloss [11].

According to the starting materials and processing techniques $\mathrm{PU} /$ silica nanocomposites are prepared by three general methods 


\title{
On the strategies for incorporating nanosilica aqueous dispersion in the synthesis of waterborne polyurethane/silica nanocomposites: Effects on morphology and properties
}

\author{
Pablo J. Peruzzo ${ }^{\mathrm{a}, *}$, Pablo S. Anbinder ${ }^{\mathrm{b}}$, Francisco M. Pardini ${ }^{\mathrm{c}}$, Oscar R. Pardini ${ }^{\mathrm{a}, \mathrm{c}}$, \\ Tomas S. Plivelic ${ }^{\mathrm{d}}$, Javier I. Amalvy ${ }^{\mathrm{a}, \mathrm{c}}$ \\ a Instituto de Investigaciones Fisicoquímicas Teóricas y Aplicadas (INIFTA: UNLP_CONICET CCT La Plata), Universidad Nacional de La Plata, Diag. 113 y \\ 64CC 16 Suc. 4, B1904DPI La Plata, Argentina \\ b Instituto de Física de Materiales Tandil (IFIMAT), CIFICEN, Consejo Nacional de Investigaciones Científicas y Técnicas (CONICET) y Universidad del Centro \\ de la Provincia de Buenos Aires, Pinto 399, B7000GHG Tandil, Argentina \\ 'Centro de Investigación y Desarrollo en Tecnología de Pinturas (CIDEPINT: CICPBA-CONICET CCT La Plata), Av. 52 e/121 y 122. B1900AYB La Plata, \\ Argentina \\ ${ }^{\mathrm{d}}$ MAX IV Laboratory, Lund University, P.O. Box 118, 22100 Lund, Sweden
}

\section{A B S T R A C T}

In this work the synthesis of waterborne polyurethane/nanosilica nanocomposites by using two different strategies is presented, starting from a vinyl terminated polyurethane prepolymer (PUP) based on isophorone diisocyanate and polypropylene glycol, and varying the nanosilica content. In one case, the PUP was dispersed in an aqueous solution containing glycerol-functionalized colloidal nanosilica particles and the dispersion was further polymerized; in the other case, the PUP was dispersed in an aqueous media, then colloidal nanosilica was added to the dispersion and then polymerized. A physical mixture was also prepared for comparative purpose. Films were prepared by casting of the aqueous dispersion.

Keywords:

Nanocomposites

Polyurethane/nanosilica

Waterborne dispersions
The morphology of the dispersions and films depended on the incorporation route of nanoparticles as was observed by SAXS, SEM and TEM. While the blends had significantly less hydrogen bonding between the hard segments of the PU and nanosilica particles, samples prepared by the two different methods proposed in this work showed an strong interaction between both materials in agreement with FTIR and DSC results. Synthesis pathway plays an important role in order to obtain high performance waterborne polyurethane/nanosilica composites, since final properties of the films also depended on the nanoparticle incorporation strategy.

\section{Introduction}

Organic/inorganic composite materials have been extensively studied for a long time. When inorganic phases in organic/inorganic composites become nanosized, they are called nanocomposites. In polymer nanocomposites the polymer chains are confined to nanoscale dimensions $(1-10 \mathrm{~nm})$. These materials have unusual properties which cannot be obtained simply by co-mixing the polymeric component with the inorganic phase at the macroscopic level [1-4]. However, the properties of nanocomposites strongly depend

* Corresponding author. Tel.: +54 $2214257291 / 7434$ (int: 115); fax: +54221 4254642

E-mail address: pjperuzzo@inifta.unl p.edu.ar (P.J. Peruzzo). on the organic matrix, inorganic nanofiller and the way in which they are prepared [5-7].

Polyurethane/silica (PU/silica) nanocomposites is one of the combinations that have attracted substantial academic and industrial interest in the last 20 years. In fact, among the numerous organic/inorganic nanocomposites, $\mathrm{PU} /$ silica nanocomposites have received much attention in recent years and they have been employed in a variety of applications [8,9]. The incorporation of nanosilica to the polyurethane matrix provide films with improved properties like increased indentation hardness, high resistance to whitening and high permeability to water vapor [10]. These materials are useful also as tough, abrasion-resistant coatings with increasing friction coefficient and reduced tackiness, with no change in gloss [11].

According to the starting materials and processing techniques $\mathrm{PU} /$ silica nanocomposites are prepared by three general methods 


\title{
On the strategies for incorporating nanosilica aqueous dispersion in the synthesis of waterborne polyurethane/silica nanocomposites: Effects on morphology and properties
}

\author{
Pablo J. Peruzzo ${ }^{\mathrm{a}, *}$, Pablo S. Anbinder ${ }^{\mathrm{b}}$, Francisco M. Pardini ${ }^{\mathrm{c}}$, Oscar R. Pardini ${ }^{\mathrm{a}, \mathrm{c}}$, \\ Tomas S. Plivelic ${ }^{\mathrm{d}}$, Javier I. Amalvy ${ }^{\mathrm{a}, \mathrm{c}}$ \\ a Instituto de Investigaciones Fisicoquímicas Teóricas y Aplicadas (INIFTA: UNLP_CONICET CCT La Plata), Universidad Nacional de La Plata, Diag. 113 y \\ 64CC 16 Suc. 4, B1904DPI La Plata, Argentina \\ b Instituto de Física de Materiales Tandil (IFIMAT), CIFICEN, Consejo Nacional de Investigaciones Científicas y Técnicas (CONICET) y Universidad del Centro \\ de la Provincia de Buenos Aires, Pinto 399, B7000GHG Tandil, Argentina \\ 'Centro de Investigación y Desarrollo en Tecnología de Pinturas (CIDEPINT: CICPBA-CONICET CCT La Plata), Av. 52 e/121 y 122. B1900AYB La Plata, \\ Argentina \\ ${ }^{\mathrm{d}}$ MAX IV Laboratory, Lund University, P.O. Box 118, 22100 Lund, Sweden
}

\section{A B S T R A C T}

In this work the synthesis of waterborne polyurethane/nanosilica nanocomposites by using two different strategies is presented, starting from a vinyl terminated polyurethane prepolymer (PUP) based on isophorone diisocyanate and polypropylene glycol, and varying the nanosilica content. In one case, the PUP was dispersed in an aqueous solution containing glycerol-functionalized colloidal nanosilica particles and the dispersion was further polymerized; in the other case, the PUP was dispersed in an aqueous media, then colloidal nanosilica was added to the dispersion and then polymerized. A physical mixture was also prepared for comparative purpose. Films were prepared by casting of the aqueous dispersion.

Keywords:

Nanocomposites

Polyurethane/nanosilica

Waterborne dispersions
The morphology of the dispersions and films depended on the incorporation route of nanoparticles as was observed by SAXS, SEM and TEM. While the blends had significantly less hydrogen bonding between the hard segments of the PU and nanosilica particles, samples prepared by the two different methods proposed in this work showed an strong interaction between both materials in agreement with FTIR and DSC results. Synthesis pathway plays an important role in order to obtain high performance waterborne polyurethane/nanosilica composites, since final properties of the films also depended on the nanoparticle incorporation strategy.

\section{Introduction}

Organic/inorganic composite materials have been extensively studied for a long time. When inorganic phases in organic/inorganic composites become nanosized, they are called nanocomposites. In polymer nanocomposites the polymer chains are confined to nanoscale dimensions $(1-10 \mathrm{~nm})$. These materials have unusual properties which cannot be obtained simply by co-mixing the polymeric component with the inorganic phase at the macroscopic level [1-4]. However, the properties of nanocomposites strongly depend

* Corresponding author. Tel.: +54 $2214257291 / 7434$ (int: 115); fax: +54221 4254642

E-mail address: pjperuzzo@inifta.unl p.edu.ar (P.J. Peruzzo). on the organic matrix, inorganic nanofiller and the way in which they are prepared [5-7].

Polyurethane/silica (PU/silica) nanocomposites is one of the combinations that have attracted substantial academic and industrial interest in the last 20 years. In fact, among the numerous organic/inorganic nanocomposites, $\mathrm{PU} /$ silica nanocomposites have received much attention in recent years and they have been employed in a variety of applications [8,9]. The incorporation of nanosilica to the polyurethane matrix provide films with improved properties like increased indentation hardness, high resistance to whitening and high permeability to water vapor [10]. These materials are useful also as tough, abrasion-resistant coatings with increasing friction coefficient and reduced tackiness, with no change in gloss [11].

According to the starting materials and processing techniques $\mathrm{PU} /$ silica nanocomposites are prepared by three general methods 


\title{
On the strategies for incorporating nanosilica aqueous dispersion in the synthesis of waterborne polyurethane/silica nanocomposites: Effects on morphology and properties
}

\author{
Pablo J. Peruzzo ${ }^{\mathrm{a}, *}$, Pablo S. Anbinder ${ }^{\mathrm{b}}$, Francisco M. Pardini ${ }^{\mathrm{c}}$, Oscar R. Pardini ${ }^{\mathrm{a}, \mathrm{c}}$, \\ Tomas S. Plivelic ${ }^{\mathrm{d}}$, Javier I. Amalvy ${ }^{\mathrm{a}, \mathrm{c}}$ \\ a Instituto de Investigaciones Fisicoquímicas Teóricas y Aplicadas (INIFTA: UNLP_CONICET CCT La Plata), Universidad Nacional de La Plata, Diag. 113 y \\ 64CC 16 Suc. 4, B1904DPI La Plata, Argentina \\ b Instituto de Física de Materiales Tandil (IFIMAT), CIFICEN, Consejo Nacional de Investigaciones Científicas y Técnicas (CONICET) y Universidad del Centro \\ de la Provincia de Buenos Aires, Pinto 399, B7000GHG Tandil, Argentina \\ 'Centro de Investigación y Desarrollo en Tecnología de Pinturas (CIDEPINT: CICPBA-CONICET CCT La Plata), Av. 52 e/121 y 122. B1900AYB La Plata, \\ Argentina \\ ${ }^{\mathrm{d}}$ MAX IV Laboratory, Lund University, P.O. Box 118, 22100 Lund, Sweden
}

\section{A B S T R A C T}

In this work the synthesis of waterborne polyurethane/nanosilica nanocomposites by using two different strategies is presented, starting from a vinyl terminated polyurethane prepolymer (PUP) based on isophorone diisocyanate and polypropylene glycol, and varying the nanosilica content. In one case, the PUP was dispersed in an aqueous solution containing glycerol-functionalized colloidal nanosilica particles and the dispersion was further polymerized; in the other case, the PUP was dispersed in an aqueous media, then colloidal nanosilica was added to the dispersion and then polymerized. A physical mixture was also prepared for comparative purpose. Films were prepared by casting of the aqueous dispersion.

Keywords:

Nanocomposites

Polyurethane/nanosilica

Waterborne dispersions
The morphology of the dispersions and films depended on the incorporation route of nanoparticles as was observed by SAXS, SEM and TEM. While the blends had significantly less hydrogen bonding between the hard segments of the PU and nanosilica particles, samples prepared by the two different methods proposed in this work showed an strong interaction between both materials in agreement with FTIR and DSC results. Synthesis pathway plays an important role in order to obtain high performance waterborne polyurethane/nanosilica composites, since final properties of the films also depended on the nanoparticle incorporation strategy.

\section{Introduction}

Organic/inorganic composite materials have been extensively studied for a long time. When inorganic phases in organic/inorganic composites become nanosized, they are called nanocomposites. In polymer nanocomposites the polymer chains are confined to nanoscale dimensions $(1-10 \mathrm{~nm})$. These materials have unusual properties which cannot be obtained simply by co-mixing the polymeric component with the inorganic phase at the macroscopic level [1-4]. However, the properties of nanocomposites strongly depend

* Corresponding author. Tel.: +54 $2214257291 / 7434$ (int: 115); fax: +54221 4254642

E-mail address: pjperuzzo@inifta.unl p.edu.ar (P.J. Peruzzo). on the organic matrix, inorganic nanofiller and the way in which they are prepared [5-7].

Polyurethane/silica (PU/silica) nanocomposites is one of the combinations that have attracted substantial academic and industrial interest in the last 20 years. In fact, among the numerous organic/inorganic nanocomposites, $\mathrm{PU} /$ silica nanocomposites have received much attention in recent years and they have been employed in a variety of applications [8,9]. The incorporation of nanosilica to the polyurethane matrix provide films with improved properties like increased indentation hardness, high resistance to whitening and high permeability to water vapor [10]. These materials are useful also as tough, abrasion-resistant coatings with increasing friction coefficient and reduced tackiness, with no change in gloss [11].

According to the starting materials and processing techniques $\mathrm{PU} /$ silica nanocomposites are prepared by three general methods 


\title{
On the strategies for incorporating nanosilica aqueous dispersion in the synthesis of waterborne polyurethane/silica nanocomposites: Effects on morphology and properties
}

\author{
Pablo J. Peruzzo ${ }^{\mathrm{a}, *}$, Pablo S. Anbinder ${ }^{\mathrm{b}}$, Francisco M. Pardini ${ }^{\mathrm{c}}$, Oscar R. Pardini ${ }^{\mathrm{a}, \mathrm{c}}$, \\ Tomas S. Plivelic ${ }^{\mathrm{d}}$, Javier I. Amalvy ${ }^{\mathrm{a}, \mathrm{c}}$ \\ a Instituto de Investigaciones Fisicoquímicas Teóricas y Aplicadas (INIFTA: UNLP_CONICET CCT La Plata), Universidad Nacional de La Plata, Diag. 113 y \\ 64CC 16 Suc. 4, B1904DPI La Plata, Argentina \\ b Instituto de Física de Materiales Tandil (IFIMAT), CIFICEN, Consejo Nacional de Investigaciones Científicas y Técnicas (CONICET) y Universidad del Centro \\ de la Provincia de Buenos Aires, Pinto 399, B7000GHG Tandil, Argentina \\ 'Centro de Investigación y Desarrollo en Tecnología de Pinturas (CIDEPINT: CICPBA-CONICET CCT La Plata), Av. 52 e/121 y 122. B1900AYB La Plata, \\ Argentina \\ ${ }^{\mathrm{d}}$ MAX IV Laboratory, Lund University, P.O. Box 118, 22100 Lund, Sweden
}

\section{A B S T R A C T}

In this work the synthesis of waterborne polyurethane/nanosilica nanocomposites by using two different strategies is presented, starting from a vinyl terminated polyurethane prepolymer (PUP) based on isophorone diisocyanate and polypropylene glycol, and varying the nanosilica content. In one case, the PUP was dispersed in an aqueous solution containing glycerol-functionalized colloidal nanosilica particles and the dispersion was further polymerized; in the other case, the PUP was dispersed in an aqueous media, then colloidal nanosilica was added to the dispersion and then polymerized. A physical mixture was also prepared for comparative purpose. Films were prepared by casting of the aqueous dispersion.

Keywords:

Nanocomposites

Polyurethane/nanosilica

Waterborne dispersions
The morphology of the dispersions and films depended on the incorporation route of nanoparticles as was observed by SAXS, SEM and TEM. While the blends had significantly less hydrogen bonding between the hard segments of the PU and nanosilica particles, samples prepared by the two different methods proposed in this work showed an strong interaction between both materials in agreement with FTIR and DSC results. Synthesis pathway plays an important role in order to obtain high performance waterborne polyurethane/nanosilica composites, since final properties of the films also depended on the nanoparticle incorporation strategy.

\section{Introduction}

Organic/inorganic composite materials have been extensively studied for a long time. When inorganic phases in organic/inorganic composites become nanosized, they are called nanocomposites. In polymer nanocomposites the polymer chains are confined to nanoscale dimensions $(1-10 \mathrm{~nm})$. These materials have unusual properties which cannot be obtained simply by co-mixing the polymeric component with the inorganic phase at the macroscopic level [1-4]. However, the properties of nanocomposites strongly depend

* Corresponding author. Tel.: +54 $2214257291 / 7434$ (int: 115); fax: +54221 4254642

E-mail address: pjperuzzo@inifta.unl p.edu.ar (P.J. Peruzzo). on the organic matrix, inorganic nanofiller and the way in which they are prepared [5-7].

Polyurethane/silica (PU/silica) nanocomposites is one of the combinations that have attracted substantial academic and industrial interest in the last 20 years. In fact, among the numerous organic/inorganic nanocomposites, $\mathrm{PU} /$ silica nanocomposites have received much attention in recent years and they have been employed in a variety of applications [8,9]. The incorporation of nanosilica to the polyurethane matrix provide films with improved properties like increased indentation hardness, high resistance to whitening and high permeability to water vapor [10]. These materials are useful also as tough, abrasion-resistant coatings with increasing friction coefficient and reduced tackiness, with no change in gloss [11].

According to the starting materials and processing techniques $\mathrm{PU} /$ silica nanocomposites are prepared by three general methods 


\title{
On the strategies for incorporating nanosilica aqueous dispersion in the synthesis of waterborne polyurethane/silica nanocomposites: Effects on morphology and properties
}

\author{
Pablo J. Peruzzo ${ }^{\mathrm{a}, *}$, Pablo S. Anbinder ${ }^{\mathrm{b}}$, Francisco M. Pardini ${ }^{\mathrm{c}}$, Oscar R. Pardini ${ }^{\mathrm{a}, \mathrm{c}}$, \\ Tomas S. Plivelic ${ }^{\mathrm{d}}$, Javier I. Amalvy ${ }^{\mathrm{a}, \mathrm{c}}$ \\ a Instituto de Investigaciones Fisicoquímicas Teóricas y Aplicadas (INIFTA: UNLP_CONICET CCT La Plata), Universidad Nacional de La Plata, Diag. 113 y \\ 64CC 16 Suc. 4, B1904DPI La Plata, Argentina \\ b Instituto de Física de Materiales Tandil (IFIMAT), CIFICEN, Consejo Nacional de Investigaciones Científicas y Técnicas (CONICET) y Universidad del Centro \\ de la Provincia de Buenos Aires, Pinto 399, B7000GHG Tandil, Argentina \\ 'Centro de Investigación y Desarrollo en Tecnología de Pinturas (CIDEPINT: CICPBA-CONICET CCT La Plata), Av. 52 e/121 y 122. B1900AYB La Plata, \\ Argentina \\ ${ }^{\mathrm{d}}$ MAX IV Laboratory, Lund University, P.O. Box 118, 22100 Lund, Sweden
}

\section{A B S T R A C T}

In this work the synthesis of waterborne polyurethane/nanosilica nanocomposites by using two different strategies is presented, starting from a vinyl terminated polyurethane prepolymer (PUP) based on isophorone diisocyanate and polypropylene glycol, and varying the nanosilica content. In one case, the PUP was dispersed in an aqueous solution containing glycerol-functionalized colloidal nanosilica particles and the dispersion was further polymerized; in the other case, the PUP was dispersed in an aqueous media, then colloidal nanosilica was added to the dispersion and then polymerized. A physical mixture was also prepared for comparative purpose. Films were prepared by casting of the aqueous dispersion.

Keywords:

Nanocomposites

Polyurethane/nanosilica

Waterborne dispersions
The morphology of the dispersions and films depended on the incorporation route of nanoparticles as was observed by SAXS, SEM and TEM. While the blends had significantly less hydrogen bonding between the hard segments of the PU and nanosilica particles, samples prepared by the two different methods proposed in this work showed an strong interaction between both materials in agreement with FTIR and DSC results. Synthesis pathway plays an important role in order to obtain high performance waterborne polyurethane/nanosilica composites, since final properties of the films also depended on the nanoparticle incorporation strategy.

\section{Introduction}

Organic/inorganic composite materials have been extensively studied for a long time. When inorganic phases in organic/inorganic composites become nanosized, they are called nanocomposites. In polymer nanocomposites the polymer chains are confined to nanoscale dimensions $(1-10 \mathrm{~nm})$. These materials have unusual properties which cannot be obtained simply by co-mixing the polymeric component with the inorganic phase at the macroscopic level [1-4]. However, the properties of nanocomposites strongly depend

* Corresponding author. Tel.: +54 $2214257291 / 7434$ (int: 115); fax: +54221 4254642

E-mail address: pjperuzzo@inifta.unl p.edu.ar (P.J. Peruzzo). on the organic matrix, inorganic nanofiller and the way in which they are prepared [5-7].

Polyurethane/silica (PU/silica) nanocomposites is one of the combinations that have attracted substantial academic and industrial interest in the last 20 years. In fact, among the numerous organic/inorganic nanocomposites, $\mathrm{PU} /$ silica nanocomposites have received much attention in recent years and they have been employed in a variety of applications [8,9]. The incorporation of nanosilica to the polyurethane matrix provide films with improved properties like increased indentation hardness, high resistance to whitening and high permeability to water vapor [10]. These materials are useful also as tough, abrasion-resistant coatings with increasing friction coefficient and reduced tackiness, with no change in gloss [11].

According to the starting materials and processing techniques $\mathrm{PU} /$ silica nanocomposites are prepared by three general methods 


\title{
On the strategies for incorporating nanosilica aqueous dispersion in the synthesis of waterborne polyurethane/silica nanocomposites: Effects on morphology and properties
}

\author{
Pablo J. Peruzzo ${ }^{\mathrm{a}, *}$, Pablo S. Anbinder ${ }^{\mathrm{b}}$, Francisco M. Pardini ${ }^{\mathrm{c}}$, Oscar R. Pardini ${ }^{\mathrm{a}, \mathrm{c}}$, \\ Tomas S. Plivelic ${ }^{\mathrm{d}}$, Javier I. Amalvy ${ }^{\mathrm{a}, \mathrm{c}}$ \\ a Instituto de Investigaciones Fisicoquímicas Teóricas y Aplicadas (INIFTA: UNLP_CONICET CCT La Plata), Universidad Nacional de La Plata, Diag. 113 y \\ 64CC 16 Suc. 4, B1904DPI La Plata, Argentina \\ b Instituto de Física de Materiales Tandil (IFIMAT), CIFICEN, Consejo Nacional de Investigaciones Científicas y Técnicas (CONICET) y Universidad del Centro \\ de la Provincia de Buenos Aires, Pinto 399, B7000GHG Tandil, Argentina \\ 'Centro de Investigación y Desarrollo en Tecnología de Pinturas (CIDEPINT: CICPBA-CONICET CCT La Plata), Av. 52 e/121 y 122. B1900AYB La Plata, \\ Argentina \\ ${ }^{\mathrm{d}}$ MAX IV Laboratory, Lund University, P.O. Box 118, 22100 Lund, Sweden
}

\section{A B S T R A C T}

In this work the synthesis of waterborne polyurethane/nanosilica nanocomposites by using two different strategies is presented, starting from a vinyl terminated polyurethane prepolymer (PUP) based on isophorone diisocyanate and polypropylene glycol, and varying the nanosilica content. In one case, the PUP was dispersed in an aqueous solution containing glycerol-functionalized colloidal nanosilica particles and the dispersion was further polymerized; in the other case, the PUP was dispersed in an aqueous media, then colloidal nanosilica was added to the dispersion and then polymerized. A physical mixture was also prepared for comparative purpose. Films were prepared by casting of the aqueous dispersion.

Keywords:

Nanocomposites

Polyurethane/nanosilica

Waterborne dispersions
The morphology of the dispersions and films depended on the incorporation route of nanoparticles as was observed by SAXS, SEM and TEM. While the blends had significantly less hydrogen bonding between the hard segments of the PU and nanosilica particles, samples prepared by the two different methods proposed in this work showed an strong interaction between both materials in agreement with FTIR and DSC results. Synthesis pathway plays an important role in order to obtain high performance waterborne polyurethane/nanosilica composites, since final properties of the films also depended on the nanoparticle incorporation strategy.

\section{Introduction}

Organic/inorganic composite materials have been extensively studied for a long time. When inorganic phases in organic/inorganic composites become nanosized, they are called nanocomposites. In polymer nanocomposites the polymer chains are confined to nanoscale dimensions $(1-10 \mathrm{~nm})$. These materials have unusual properties which cannot be obtained simply by co-mixing the polymeric component with the inorganic phase at the macroscopic level [1-4]. However, the properties of nanocomposites strongly depend

* Corresponding author. Tel.: +54 $2214257291 / 7434$ (int: 115); fax: +54221 4254642

E-mail address: pjperuzzo@inifta.unl p.edu.ar (P.J. Peruzzo). on the organic matrix, inorganic nanofiller and the way in which they are prepared [5-7].

Polyurethane/silica (PU/silica) nanocomposites is one of the combinations that have attracted substantial academic and industrial interest in the last 20 years. In fact, among the numerous organic/inorganic nanocomposites, $\mathrm{PU} /$ silica nanocomposites have received much attention in recent years and they have been employed in a variety of applications [8,9]. The incorporation of nanosilica to the polyurethane matrix provide films with improved properties like increased indentation hardness, high resistance to whitening and high permeability to water vapor [10]. These materials are useful also as tough, abrasion-resistant coatings with increasing friction coefficient and reduced tackiness, with no change in gloss [11].

According to the starting materials and processing techniques $\mathrm{PU} /$ silica nanocomposites are prepared by three general methods 